Med Klin Intensivmed Notfmed 2021 - 116: 375-376

https://doi.org/10.1007/s00063-021-00809-0

Angenommen: 5. März 2021

(c) Springer Medizin Verlag GmbH, ein Teil von Springer Nature 2021

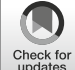

Check for
updates

Die Versorgung des polytraumatisierten Schockpatienten ist seit vielen Jahren klar strukturiert $[1,2]$. Sowohl im Weißbuch Schwerverletztenversorgung als auch in der S3-Leitlinie Polytrauma/ Schwerverletztenbehandlung wird auf die Versorgung von polytraumatisierten Schockpatienten im Detail eingegangen $[1,2]$.

Die meisten Patienten, die im Schockraum versorgt werden, weisen jedoch kardiovaskuläre (33\%) und zentralnervöse $(21 \%)$ Krankheitsbilder auf [3]. Weitere Gründe waren respiratorisch (13\%), gastrointestinal (9\%), renal $(8 \%)$, septisch $(7 \%)$ und - eher selten traumatologisch (9\%) bedingt. In einer ersten nationalen Studie zum Schockraummanagement (OBSERvE-Studie, „observation of critically ill patients in the resuscitation room of the emergency department") konnte gezeigt werden, dass nichttraumatologische Schockraumpatienten beruhend auf einer kardiovaskulären Genese mit $32 \%$ den größten Anteil ausmachen; $31 \%$ wiesen eine zentralnervöse, $18 \%$ eine pulmonale, $10,2 \%$ eine septische und $4,3 \%$ eine abdominelle Ursache auf [4]. Beruhend auf dieser studienbasierten Evidenz, dass der Anteil der nichttraumatologischen Schockraumpatienten weitaus über dem der traumatologischen Patienten liegt, ist es verwunderlich, warum bis dato keine Empfehlungen zum nichttraumatologischen Schockraummanagement ausgesprochen wurden.

Das Management von traumatologischen und nichttraumatologischen Schockraumpatienten setzt ein trainiertes Personal voraus [5]. Das Teamtraining sollte hier an erster Stelle stehen. Daher

\title{
Guido Michels
}

Klinik für Akut- und Notfallmedizin, St.-Antonius-Hospital gGmbH, Akademisches Lehrkrankenhaus der RWTH Aachen, Eschweiler, Deutschland

\section{Schockraummanagement}

\section{Heute und morgen}

bilden ein ATLS $^{\circledR}$ (Advanced Trauma Life Support)-Kurs im Rahmen des Traumabzw. ein ACILS $^{\circledR}$ (Advanced Critical Illness Life Support)-Kurs im Rahmen des Nichttraumaschockraummanagements, der von der Deutschen Gesellschaft Interdisziplinäre Notfall- und Akutmedizin e. V. (DGINA) zeitnah eingeführt wird, die Basis einer qualitativen Versorgung von Schockraumpatienten. Nur durch ein kontinuierlich gelebtes interprofessionelles und interdisziplinäres Schockraumteamtraining ist erst ein optimales Schockmanagement möglich. Eine interprofessionelle und interdisziplinäre Teamarbeit ist dabei für die Patientenversorgung und die Patientensicherheit unerlässlich. Da ungefähr $60 \%$ aller Fehler in der Medizin ursächlich dem Bereich der „human factors“ zugeordnet werden, sollte das Teamtraining auch auf das sog. Crew Resource Management (CRM) eingehen. C. Kersten und Koautoren gehen in ihrem Beitrag „Crew Resource Management im Schockraum“ auf die theoretischen und praktischen CRM-Prinzipien sowie CRM-Trainingskonzepte ein.

Bei Traumapatienten gehört die Notfallsonographie bzw. die eFAST-Sonographie („extended focused asessment with sonography in trauma") zum festen Bestandteil des Schockraummanagements [1, 6]. Anhand der fokussierten Notfallsonographie können viele Akutdiagnosen im Schockraum sicher und schnell diagnostiziert werden. Aus diesem Grund sollte die Notfallsonographie als bettseitige Bildgebung standardisiert im Schockraummanagement insbesondere bei Nichttraumapatienten integriert werden [7]. Im Rahmen der Zusatzwei- terbildung Klinische Akut-und Notfallmedizin wird daher auch die Notfallsonographie von Abdomen, Thorax, Herz, Gefäßen und Bewegungsapparat als eines von vielen notfallmedizinischen Kernverfahren aufgeführt. D. Hempel unterstreicht in ihrem Beitrag „Fokussierte Sonographie im Schockraum“, dass ein strukturiertes Vorgehen gerade im Schockraum aufgrund der dynamischen und zeitkritischen Situation absolut notwendig ist.

Die Schwerpunkte „Trauma- und Nichttraumaschockraummanagement " werden von den Erstautoren V. Ketter und M. Michael präsentiert. V. Ketter und Koautoren stellen den aktuellen Stand zum „Traumatologischen Schockraummanagement" vor. In ihrem Beitrag gehen die Autoren auf die Schockraumaktivierung und -organisation, die Basisdiagnostik im Schockraum und auf verschiedene Verletzungsmuster ein. M. Michael und Autoren beschreiben in ihrem Beitrag „Nichttraumatologisches Schockraummanagement" auf der Basis von jüngst publizierten Studienergebnissen eine strukturierte Vorgehensweise im nichttraumatologischen Schockraummanagement. Die gemeinsame Entwicklung von Empfehlungen zum nichttraumatologischen Schockraummanagement unter Einbeziehung aller an der Notfallversorgung beteiligten Fachdisziplinen wird eine Herausforderung in Zukunft darstellen.

Liebe Leserinnen und Leser, ich hoffe, dass Sie viele wertvolle Informationen und praktische Hinweise für Ihren Alltag in der Akutmedizin mitnehmen können. An dieser Stelle bedanke ich mich sehr bei allen Autorinnen und Autoren für 
ihren Einsatz und die hochqualitativen Beiträge in dieser Kongressausgabe 2021 der Zeitschrift Medizinische Klinik - Intensivmedizin und Notfallmedizin.

Ihr

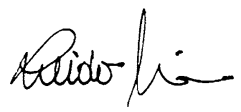

Guido Michels

\section{Korrespondenzadresse}

Prof. Dr. med. Guido Michels

Klinik für Akut- und Notfallmedizin, St.Antonius-Hospital gGmbH, Akademisches Lehrkrankenhaus der RWTH Aachen Dechant-Deckers-Str. 8, 52249 Eschweiler, Deutschland

guido.michels@sah-eschweiler.de

Interessenkonflikt. G. Michels gibt an, dass kein Interessenkonflikt besteht. G. Michels ist Sprecher der Arbeitsgruppe Kardiopulmonale Reanimation und des Arbeitskreises Mechanische Kreislaufunterstützung der Arbeitsgruppe Interventionelle Kardiologie der Deutschen Gesellschaft für Kardiologie e. V. (DGK) und erhielt Honorare für Vortragstätigkeiten von Zoll, Getinge, Sedana Medical und Orion Pharma. Die Interessenkonflikte stehen in keinem Zusammenhang mit der vorliegenden Arbeit.

\section{Literatur}

1. Deutsche Gesellschaft für Unfallchirurgie (2019) Weißbuch Schwerverletztenversorgung. Empfehlungen zur Struktur, Organisation und Ausstattung sowie Forderung von Qualität und Sicherheit in der Schwerverletztenversorgung in der BundesrepublikDeutschland, 3. Aufl.

2. https://www.awmf.org/uploads/tx_szleitlinien/ 012-019| S3 Polytrauma SchwerverletztenBehandlung_2017-08.pdf;. Zugegriffen: 1. März 2021

3. Piagnerelli $M$, Van Nuffelen $M$, Maetens $Y$, Lheureux P, Vincent JL (2009) A 'shock room' for early management of the acutely ill. Anaesth Intensive Care 37(3):426-431

4. Bernhard M, Döll S, Hartwig T et al (2018) Resuscitation room management of critically ill nontraumatic patients in a German emergency department (OBSERvE-study). Eur J Emerg Med 25(4):e9-e17

5. Tiel Groenestege-Kreb D, van Maarseveen O, Leenen L (2014) Trauma team. Br J Anaesth 113(2):258-265

6. Wongwaisayawan S, Suwannanon R, Prachanukool T, Sricharoen P, Saksobhavivat N, Kaewlai R (2015) Trauma ultrasound. Ultrasound Med Biol 41(10):2543-2561

7. Michels G, Zinke H, Möckel M et al (2017) Recommendations for education in ultrasound in medical intensive care and emergency medicine: position paper ofDGIIN, DEGUM and DGK. Med Klin Intensivmed Notfmed 112(4):314-319

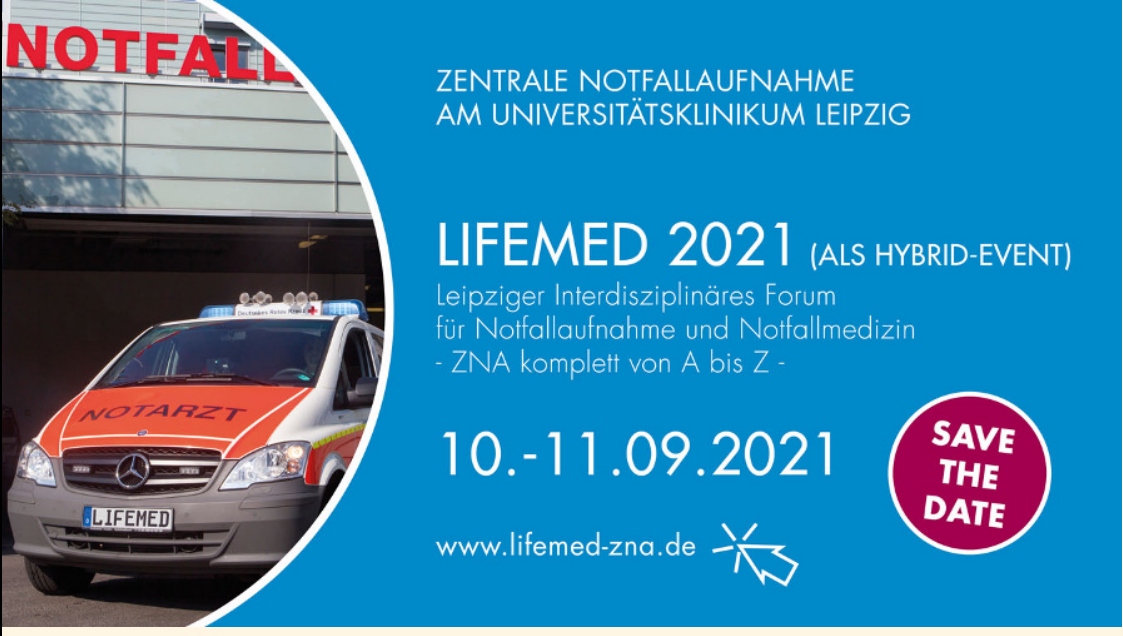

\section{LIFEMED 2021 - 10.09. und 11.09.2021 in Leipzig}

Das diesjähriges LIFEMED Symposium als Hybridveranstaltung. Das Organisationsteam ist optimistisch, dass dieses Format sowohl vor dem Hintergrund der dann aktuellen Lage mit regelmäßigen Testmöglichkeiten und einer höheren Impfquote umsetzbar ist und so den notfallmedizinisch interessierten Kolleginnen und Kollegen aller Berufsgruppen und Fachbereiche unter Einhaltung der Hygieneregeln endlich wieder die Möglichkeit gibt, sich auch direkt vor Ort auch im persönlichen Austausch fort- und weiterzubilden.

\section{Freitag, 10.09.2021 Vortragspro-}

gramm: Neben Themen wie "COVID-19 und Zentrale Notaufnahme - was haben wir gelernt?", „Aktuelle Konzepte, Strukturen und politische Entwicklungen" stehen UpdateVeranstaltungen u.a. zu Kindernotfällen, zur Versorgung geriatrischer Notfallpatienten, zu psychiatrischen Notfällen und zum Polytraumamanagement auf dem Programm. Für die einzelnen Sitzungen ist ein ganz neues Format vorgesehen: Die Präsentationen erfolgen in Dialogform bzw. als Pro- und Con-Vorträge. Die Update-Sitzungen orientieren sich jeweils an einem konkreten Fall liefern zur Thematik aber auch den aktuellen wissenschaftlichen Hintergrund. In Zusammenarbeit mit dem DRK Sachsen findet nachmittags der diesjährige LIFEMED-Rettungsdienst-Workshop statt.
Samstag, 11.09.2021 Workshops: Dabei werden intraossärer Zugang, Thoraxdrainage und Koniotomie in einem gemeinsamen Workshop Invasive Notfalltechniken angeboten. Der Workshop Notfallmedizin kompakt wird für Berufseinsteiger aber auch als Refresherkurs in bewährter Form die verschiedenen Themen rund um die Notaufnahme aufgreifen und kompakt anbieten. Als besonderes Highlight wird im Rahmen des erstmalig bei LIFEMED 2021 angebotenen VR-Workshop ein Notfallmedizinisches Training an realitätsnahen virtuellen Patienten, losgelöst von räumlichen Gegebenheiten und Limitierungen im Team in Echtzeitkommunikation und -kooperation möglich sein.

LIFEMED 2021 findet genau 20 Jahre nach den Angriffen auf das World Trade Center in New York 2001, 9/11 statt. Zur Erinnerung an diese Anschläge wird eine zu dieser Zeit im Emergency Department des Mount Sinai Hospital in Manhattan tätige Kollegin, für einen Vortrag zu den Erfahrungen dieser Zeit und der anschließenden Bewältigung berichten.

Last but not least wird es wieder ein GetTogether geben: Nach Bootstransfer auf den Leipziger Kanälen möchten in spannender und interessanter Umgebung wieder die Gelegenheit zum direkten Dialog mit Referenten, Teilnehmern und Kollegen geben.

\section{Anmeldung und Information:}

www.ukl-live.de/lifemed 